\title{
Group and Renormgroup Symmetry of Quasi-Chaplygin Media
}

\author{
Vladimir F. KOVALEV \\ Institute for Mathematical Modelling, Miusskaya 4-A, \\ Moscow, 125047, RUSSIA \\ e-mail: kovalev\#9@imamod.msk.su
}

\begin{abstract}
Results of renormgroup analysis of a quasi-Chaplygin system of equations are presented. Lie-Bäcklund symmetries and corresponding invariant solutions for different "Chaplygin" functions are obtained. The algorithm of construction of a group on a solution (renormgroup) using two different approaches is discussed.
\end{abstract}

The report is devoted to a group and renormgroup analysis of quasi-Chaplygin system of equations. By this we mean the system of two first-order partial differential equations for $v$ and $n$

$$
\begin{aligned}
& v_{t}+v v_{x}=a \varphi(n) n_{x}, \quad n_{t}+v n_{x}+n v_{x}=0, \\
& 0 \leq n<+\infty, \quad-\infty<v<+\infty
\end{aligned}
$$

with constant $a$ and a nonlinearity function $\varphi$ which depends only on $n$. Despite of the simple form, this mathematical model has a wide field of applications. By means of the hodograph transformation this system of equations is reduced to the system of partial differential equations (PDE) for two functions $\tau=n t$ and $\chi=x-v t$ depending on "velocity" $v$ and "density" $n$

$$
\tau_{v}-\psi(n) \chi_{n}=0, \quad \chi_{v}+\tau_{n}=0, \quad a \varphi \psi=n .
$$

Equations (2) were first treated in gas dynamics by Chaplygin in 1902 (see also [1]) and the function $(1 / \psi)$ is referred to by his name. The progress in nonlinear optics and plasma theory made it evident that the field of application of mathematical model based on equations (1) (and therefore (2)) goes far beyond gas dynamics. To characterize the media which exhibit the behavior similar to that of a Chaplygin gas, the term "quasiChaplygin media" was suggested. Examples of physical phenomena that take place in different quasi-Chaplygin media described by PDE (1) with various nonlinearities $\varphi(n)$ were recently discussed in [2]. It should be noted that the physical meaning of variables $t, x, v$ and $n$ may differ for different problems from that in gas dynamics. For example, in nonlinear geometrical optics, $t$ and $x$ are coordinates respectively along and transverse to the direction of laser-beam propagation, $v$ is the derivative of eikonal with respect to $x$ and $n$ is a laser beam intensity. 
The use of a symmetry group admitted by PDE under consideration enables to find exact analytical solutions of boundary-value problems for systems (1) and (2) that are both of theoretical and experimental interest. For example, in view of the nonlinearity function

$$
\varphi(n)=n(n+b)^{c}
$$

these solutions describe the self-focusing of a laser beam in a nonlinear medium, the filamentation of an electron beam in plasma, etc.

The s-th order Lie-Bäcklund (L-B) symmetry of system (2) is characterized by a canonical group generator (see, for example, [3])

$$
X=f \partial_{\tau}+g \partial_{\chi},
$$

where coordinates $f$ and $g$ appear to be linear in $\tau, \chi$ and their $s$ derivatives with respect to $n$

$$
\begin{aligned}
& f=\bar{f}+\sum_{i=0}^{s}\left(a^{i} \tau_{i}+b^{i} \chi_{i}\right), \quad g=\bar{g}+\sum_{i=0}^{s}\left(\alpha^{i} \tau_{i}+\beta^{i} \chi_{i}\right) \\
& \tau_{s}=\left(\partial^{s} \tau / \partial n^{s}\right), \quad \chi_{s}=\left(\partial^{s} \chi / \partial n^{s}\right) .
\end{aligned}
$$

All coefficients in linear forms (5) are functions of two variables $v, n$ and the feasible parameters of the system (2) (in case of nonlinearity (3) these are $a, b$ and $c$ ).

First-order $(s=1)$ L-B symmetries are equivalent to the Lie point symmetries obtained earlier in [4] (see also [3], p.92) ${ }^{\dagger}$. For arbitrary nonlinearities $\varphi(n)$ the symmetry Lie algebra is infinite and is spanned by operators of dilatations of $\tau$ and $\chi$ and translations along the $v$-axis and an infinite-dimensional ideal described by group the generator $X_{\infty}$ with coordinates $\bar{f}, \bar{g}$ which are arbitrary solutions of linear PDE (2). System (2) admits two additional operators iff the function $\psi(n)$ satisfies the equation

$$
\psi_{1}\left(\psi / \psi_{1}\right)_{n n} \mp \omega^{2}=0, \quad \psi_{1} \neq 0, \quad \omega^{2}=\text { const . }
$$

It is easily checked that for the function $\varphi$ of special form (3) equation (6) is satisfied for $\omega^{2}=0$. Moreover, if we consider the transformations of parameters $a, b$ and $c$ along with transformations of dynamic variables, we obtain in the latter case two more group generators [5], [6] (the parameter $c$ is a group invariant). These generators are of special interest to construct a group on a solution which is discussed later.

As for L-B symmetries of high order $(s \geq 2)$, the system (2) admits a single group generator of the given order $s$ and transformations between symmetries of different orders are governed by one recursion operator. This result is valid for the nonlinearity function $\varphi(n)$ of a general form, and L-B generators thus found correlate with those obtained in [7]. In case of the nonlinearity function of the special form (6), we obtain the following result: as the order $s$ of L-B symmetry increases, so does the number of corresponding group generators: for $s=2$ we obtain five group generators, while for $s=3$ there are seven group generators and so on [5]. Transformations between these symmetries are obtained with the help of three recursion operators.

\footnotetext{
${ }^{\dagger}$ The problem was also discussed in details by S.V. Meleshko (Isentropical Flow of an Ideal Gas, 1989, unpublished).
} 
A procedure of finding L-B symmetries involves tedious computations that become rather complicated for high values of the order $s$ and are unlikely to be done by hand. To overcome these difficulties, one can use symbolic programs for determination of symmetry groups of systems of differential equations. We have used the program DIMSYM [8] that is based on the computer algebra system REDUCE. An attractive feature of this package is that along with a set of heuristic rules for integration of a system of determining equations it also uses a heuristic-free algorithm of reduction of these equations to a standard (involutive) form. In our case this way of solution appears to be the most efficient, especially when calculation of integrability conditions is complemented with direct integration and simplification (expert regime and this procedure for $s=2$ will be described in details elsewhere). Having the idea of symmetry calculation for $s=2$ in mind, we succeeded using the DIMSYM package to calculate high-order $(s=2,3,4)$ L-B symmetries in a rational time, yet in combination with some manual manipulations. In view of the linear form representation for coordinates $f$ and $g$ given by formulae (5), this time is drastically reduced.

Using L-B symmetries obtained, we can calculate invariant solutions that obey both basic equations (2) and invariance conditions

$$
f=0, \quad g=0
$$

which are taken in the form of a linear combination of coordinates of the Lie point and second-order L-B group generators. We present here three invariant solutions of system (2) with nonlinearity function (3) for different values of the parameter $c$ and $b=0$ :

Example 1: $c=-1$;

$$
\begin{aligned}
& \tau=\frac{(v / 2)^{1 / 2}}{a^{3 / 4}}\left(\sqrt{\kappa^{2}+1}-\kappa\right)^{1 / 2}, \quad \kappa=\frac{\sqrt{a}}{v}\left(1-n-\frac{v^{2}}{4 a}\right), \\
& \chi=-\frac{1}{2} \ln \frac{(v / 2 \sqrt{a})^{1 / 2}+\left(\sqrt{\kappa^{2}+1}-\kappa\right)^{1 / 2}}{-(v / 2 \sqrt{a})^{1 / 2}+\left(\sqrt{\kappa^{2}+1}-\kappa\right)^{1 / 2}} .
\end{aligned}
$$

Example 2: $c=-3 / 2$;

$$
\begin{aligned}
& \tau=\frac{v^{3 / 2}}{a^{5 / 4}}\left(\sqrt{\kappa^{2}+1}-\kappa\right)^{1 / 2}\left(\sqrt{\kappa^{2}+1}+2 \kappa\right), \quad \kappa=\frac{2 \sqrt{a}}{v}\left(1-\sqrt{n}-\frac{v^{2}}{16 a}\right), \\
& \chi=3 \ln \frac{(v / \sqrt{a})^{1 / 2}+2\left(\sqrt{\kappa^{2}+1}-\kappa\right)^{1 / 2}}{-(v / \sqrt{a})^{1 / 2}+2\left(\sqrt{\kappa^{2}+1}-\kappa\right)^{1 / 2}}+3\left(\frac{v}{\sqrt{a}}\right)^{1 / 2}\left(\sqrt{\kappa^{2}+1}-\kappa\right)^{1 / 2} \times \\
& \left(\sqrt{\kappa^{2}+1}+\kappa-\frac{v}{4 \sqrt{a}}\right)\left(\frac{v^{2}}{16 a}+\frac{\kappa v}{2 \sqrt{a}}-1\right)^{-1} .
\end{aligned}
$$

Example 3: $c=-3$;

$$
\tau=\frac{a^{1 / 4}}{4 v^{3 / 2}}\left(\sqrt{\kappa^{2}+1}-\kappa\right)^{1 / 2} \frac{\left(\sqrt{\kappa^{2}+1}+2 \kappa\right)}{\left(\kappa^{2}+1\right)^{3 / 2}}, \quad \kappa=\frac{\sqrt{a}}{v}\left(\frac{1}{n}-1+\frac{v^{2}}{4 a}\right),
$$




$$
\begin{aligned}
& \chi=-\frac{1}{4}\left(\frac{\sqrt{a}}{v}\right)^{3 / 2}\left(\sqrt{\kappa^{2}+1}-\kappa\right)^{1 / 2}\left[\frac{3}{4}\left(\kappa^{2}+1\right)^{-1 / 2}\left(\frac{v^{2}}{a}+\frac{4}{3}\left(1-\kappa \frac{v}{\sqrt{a}}\right)\right)-\right. \\
& \left.2 \frac{v}{\sqrt{a}}+\frac{\sqrt{\kappa^{2}+1}+2 \kappa}{8\left(\kappa^{2}+1\right)^{3 / 2}}\left(-\frac{v^{3}}{a^{3 / 2}}+4 \frac{v}{\sqrt{a}}\left(1-2 \kappa^{2}\right)+6 \kappa\left(\frac{v^{2}}{a}-\frac{4}{3}\right)\right)\right] .
\end{aligned}
$$

It should be pointed out that solution (8) was found by Akhmanov et al. [9] about 30 years ago. Our approach [10] reveals the group-theoretical nature of this result.

The group generators obtained characterize the symmetry of the basic differential manifold. However, these generators enable to construct such a symmetry group that describes not only the symmetry of basic equations but the symmetry of a solution of a boundary-value problem as well. We call this symmetry group a group on a solution of a boundary-value problem, which thus appears as an invariant manifold of this group generator. In the context of the general approach developed by D.V.Shirkov [11], this group can be referred to as a renormalization group ( $R G)$, of course in a narrow sense.

In order to construct the group on a solution, we can use two different algorithms. The first one is based on the restriction of a point symmetry group, more precisely its infinite dimensional ideal, admitted by the original differential equations, on the solution of the boundary-value problem [12], [13]. The second way of construction of a group on a solution is based on a point-symmetry group analysis for a system of basic differential equations and additional differential constraint that explicitly takes the boundary data into account [13]. Such a constraint may result for example from an appropriate coordinate of a canonical operator of the Lie-Bäcklund group, when the latter vanishes.

We demonstrate the first algorithm of RG construction for equations (2) with the nonlinearity function $\psi(n)$ of the special form (3). The Lie point group admitted by these equations is characterized by generators $X_{i}, i=1, \cdots, 6$ and $X_{\infty}$

$$
X=X_{\infty}+\sum_{i=1}^{6} c_{i} X_{i} \equiv\left(\bar{f}+\sum_{i=1}^{6} c_{i} f_{i}\right) \partial_{\tau}+\left(\bar{g}+\sum_{i=1}^{6} c_{i} g_{i}\right) \partial_{\chi}
$$

Restriction on the solution means that coordinates $f$ and $g$ of the canonical operator (11) turn to zero on the solution of the boundary-value problem

$$
\bar{f}=-\sum_{i=1}^{6} c_{i} f_{i}, \quad \bar{g}=-\sum_{i=1}^{6} c_{i} g_{i} .
$$

In contrast to (7) formulae (12) should be treated not as equations for $\tau$ and $\chi$ but as relationships that yield expressions for the functions $\bar{f}$ and $\bar{g}$ in terms of $f_{i}, g_{i}, i=1, \cdots, 6$ taken on the solution $\tau=\bar{\tau}(v, n), \chi=\bar{\chi}(v, n)$ of a boundary-value problem. Substitution of (12) into (11) gives six renormgroup operators

$$
R=\sum_{i=1}^{6} c_{i}(a, b) R_{i}
$$

each being determined by corresponding coordinates $f_{i}, g_{i}$ and a pair of functions $A^{i}, B^{i}$ explicitly defined by the solution $\bar{\tau}(v, n), \bar{\chi}(v, n)$. As an example, we present here only 
one renormgroup operator $R_{5}$

$$
\begin{aligned}
& R_{5}=\left(-\frac{\tau}{c+2}-A^{5}\right) \partial_{\tau}-B^{5} \partial_{\chi}-\frac{n+b}{c+2} \partial_{n}+a \partial_{a}, \\
& A^{5}=-a \bar{\tau}_{a}-\frac{1}{c+2}\left(\bar{\tau}-(n+b) \bar{\tau}_{n}\right), \quad B^{5}=-a \bar{\chi}_{a}+\frac{n+b}{c+2} \bar{\chi}_{n} .
\end{aligned}
$$

For the solution described by formulae (8), functions $A^{5}, B^{5}$ in (14) are expressed as follows:

$$
\begin{aligned}
& A^{5}=-\frac{(v / 2)^{1 / 2}}{4 a^{3 / 4} \sqrt{1+\kappa^{2}}}\left(\sqrt{\kappa^{2}+1}-\kappa\right)^{1 / 2}\left(\kappa+\sqrt{1+\kappa^{2}}-2 \frac{\sqrt{a}}{v}\right), \\
& B^{5}=\frac{(v / 2)^{1 / 2}}{4 a^{1 / 4} \sqrt{1+\kappa^{2}}} \frac{\left(\sqrt{\kappa^{2}+1}-\kappa\right)^{1 / 2}}{\left(\sqrt{\kappa^{2}+1}-\kappa-(v / 2 \sqrt{a})\right)}\left(\sqrt{1+\kappa^{2}}-3 \kappa+2 \frac{\sqrt{a}}{v}-\frac{v}{\sqrt{a}}\right) .
\end{aligned}
$$

Now we demonstrate the second algorithm of RG construction for the boundaryvalue problem with the help of differential constraints. The number of distinct differential constraints that satisfy given boundary data is infinite, so is the number of various RGs. To be specific, we assume the latter in the form of the linear combination (7) of coordinates of L-B second-order group generators with $b=0$ and $c=-1$ which correspond to boundary conditions for the solution (8):

$$
\begin{aligned}
& \left(2 n(1-n)+\left(n v^{2} / 2 a\right)\right) \tau_{n n}-n \tau_{n}-\left(2 n^{2} v / a\right) \chi_{n n}-(2 n v / a) \chi_{n}=0, \\
& 2 v n \tau_{n n}+v \tau_{n}+\left(2 n(1-n)+\left(n v^{2} / 2 a\right)\right) \chi_{n n}+\left(2-3 n+\left(n v^{2} / 2 a\right)\right) \chi_{n}=0 .
\end{aligned}
$$

Group analysis of (2) and (15) and subsequent restriction of the point group obtained on the perturbation theory solution of the boundary-value problem (in the sense of the equality $f=0, g=0$ similar to the relationship (12)) yield two RG operators

$$
\bar{R}_{1}=(\tau-\bar{\tau}(v, n)) \partial_{\tau}+(\chi-\bar{\chi}(v, n)) \partial_{\chi}, \quad \bar{R}_{2}=\tau \partial_{\tau}-v \partial_{v}-2 a \partial_{a},
$$

where functions $\bar{\tau}(v, n)$ and $\bar{\chi}(v, n)$ are given by the right-hand sides of expressions (8). The first operator in (16) coincides with $R_{1}$ from the sum (13), while the second operator $\bar{R}_{2}$ describes the "trivial" symmetry of a solution.

\section{References}

[1] Chaplygin S.A., On gas jets, GITTL, Moscow-Leningrad, 1949.

[2] Trubnikov B.A. and Zhdanov S.K., Quasi-Gaseous Unstable Media, Nauka Publishers, Moscow, 1991.

[3] CRC Handbook of Lie Group Analysis of Differential Equations, V.1, Symmetries, Exact Solutions and Conservation Laws, ed. by N.H. Ibragimov CRC Press, Boca Raton, Florida, USA, 1994.

[4] Ovsyannikov L.V., Group Properties of Differential Equations, Izdat Sibirsk. Otdel. Akad. Nauk SSSR, Novosibirsk, 1962, p.136.

[5] Kovalev V.F., Pustovalov V.V. and Senashov S.I., Differential Equations, 1993, V.29, 1521. 
[6] Pustovalov V.V. and Shvarev A.K., Group and Renormgroup Analysis of Nonlinear Geometrical Optics Equations, 1. Lie symmetry, P.N. Lebedev Physical Institute, Preprint N 15, April 1994.

[7] Sheftel M.B., Vestnik LGU, 1982, N 7, 37; Theor. i Matem. Fiz., 1983, V.56, 368.

[8] Sherring J. and Prince G., DIMSYM - Symmetry Determination and Linear Differential Equations Package, Department of Mathematics, La Trobe University, Melbourne, Australia, preprint 1992.

[9] Akhmanov S.A., Khokhlov R.V. and Sukhorukov A.P., ZhETF, 1966, V.50, 1537.

[10] Kovalev V.F. and Pustovalov V.V., Quasi-Chaplygin Media Equations: Theoretical Group Approach, Institute for Mathematical Modelling, Preprint N 14, 1994.

[11] Shirkov D.V., Usp. Matem. Nauk, 1994, V.49, 147-164.

[12] Kovalev V.F. and Pustovalov V.V., Lie Groups and their Applications, 1994, V.1, N 2, 104 (Celal Bayar University, Manisa, Turkey).

[13] Kovalev V.F., Krivenko S.V. and Pustovalov V.V., Lie Symmetry and a Group on a Solution of a Boundary-Value Problem, P.N.Lebedev Physical Institute, Preprint N 13, April 1995. 